\title{
Lifetime risk of suicide in people with schizophrenia lower than commonly reported
}

Palmer BA, Pankratz VS, Bostwick JM. The lifetime risk of suicide in schizophrenia: a reexamination. Arch Gen Psychiatry 2005;62:247-53.

\section{What is the lifetime risk of suicide in people diagnosed with schizophrenia?}

\section{METHODS}

-

Design: Systematic review of cohort studies with meta-analysis.

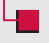

Data sources: MEDLINE (from 1966), PsycINFO (from 1984),

plus hand search of reference lists, papers, and textbooks

covering affective disorder. Search date not specified.

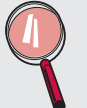

Study selection and analysis: Cohort studies were included if they involved people diagnosed with schizophrenia, had a follow up of at least two years, and did not lose more than $10 \%$ of participants to follow up. Studies limited to a particular age group or in people diagnosed with schizoaffective disorders were excluded. Studies enrolling people at the onset of schizophrenia or first admission for schizophrenia were analysed separately as they cover the initial period of illness when suicide risk is highest, and therefore provide a more accurate estimate of lifetime suicide risk. Case fatality rates (proportion of cohort (alive and dead) that committed suicide) and proportionate mortality rates (proportion of deaths in the cohort that were due to suicide) were determined for each study. Regression models were used to calculate the point where case fatality rate and proportionate mortality rate were equal: a theoretical point when all subjects have died which provided an estimate of lifetime suicide risk.

Outcomes: Lifetime risk of suicide.

\section{MAIN RESULTS}

Twenty nine cohort studies $(\mathrm{n}=22598)$ met inclusion criteria and followed people from the onset of schizophrenia or first admission for schizophrenia. The estimated lifetime suicide risk in this population was $5.6 \%$ (95\% CI $3.7 \%$ to $8.5 \%)$.

\section{CONCLUSIONS}

Using meta-analysis combining case fatality and proportionate mortality rate data, the estimated lifetime risk of suicide for first admission and new-onset cases of schizophrenia is $5.6 \%$. This estimate is lower than the widely reported estimate of $10 \%$.

\section{NOTES}

As individual patient data for each cohort were unavailable, preferred survival analysis techniques such as Kaplan-Meier could not be used.

For correspondence: John Michael Bostwick, MD, Department of Psychiatry and Psychology, Mayo Clinic, 200 First Street SW, Rochester, MN 55905, USA; john@mayo.edu

Source of funding: American Foundation for Suicide Prevention.
The method of analysis used by this paper addresses the shortcomings of using either proportionate mortality rate or case fatality rate alone to estimate lifetime suicide risk. The proportionate mortality rate overestimates the risk of suicide, particularly in studies with a short follow up, as it assumes that suicides occur at the same rate over time relative to the total number of deaths. The case fatality rate may underestimate suicide prevalence as some suicides will inevitably be missed if not all subjects are followed up until they die.

\section{Commentary}

- or several decades, the literature has quoted the lifetime risk of suicide in schizophrenia as $10-15 \%$. The most frequently cited review is that of Miles et al who examined mortality reports published between 1931 and 1975, and estimated the lifetime risk to be $10 \%{ }^{1}$ This figure was subsequently challenged by Inskip et al who analysed 29 studies using proportionate mortality (the percentage of the dead who died by suicide) and estimated the risk to be substantially lower at $4 \%{ }^{2}$

The systematic review by Palmer et al provides the most accurate estimate to date of the risk of suicide in schizophrenia. Their metaanalysis uses both proportionate mortality and case fatality (the percentage of the original sample who died by suicide) and would be expected to provide a better estimate of risk, given that many suicides occur early in the course of the illness (estimates based only on proportionate mortality run the risk of overestimating suicide risk). Palmer et al also reviewed studies of first episode and first admission patients and found that for these cohorts the lifetime risk of suicide was higher than for studies of allcomers. Patients with schizoaffective disorder, in whom the risk of suicide is thought to be particularly high, ${ }^{3}$ were excluded.

The estimate of lifetime risk of suicide in schizophrenia is thus lower than was formerly thought, with greatest risk in the first few years of illness. This information, together with knowledge of risk factors for suicide in schizophrenia (previous depression, previous suicide attempts, drug misuse, agitation, fear of mental disintegration, poor adherence to treatment, and recent loss), ${ }^{4}$ should help mental health services focus on those at highest risk. Targeting of affective symptoms, substance misuse, and compliance, particularly in the first few years of the illness, would appear to be important in suicide prevention.

Camilla M Haw MRCPsych Centre for Suicide Research, University of Oxford, Oxford, UK

1 Miles CP. Conditions predisposing to suicide: a review. J Nerv Ment Dis 1977; 164:231-46.

2 Inskip HM, Harris C, Barraclough B. Lifetime risk of suicide for affective disorder, alcoholism and schizophrenia. Br J Psychiatry 1998:172:35-7.

3 Fenton WS, McGlashan TH, Victor BJ, et al. Symptoms, subtype and suicidality in patients with schizophrenia spectrum disorders. Am J Psychiatry 1997;154:199-204.

4 Hawton K, Sutton S, Haw C, et al. Schizophrenia and suicide: a systematic review of risk factors. Br J Psychiatry 2005;187:8-20. 